\title{
Anterior elongation of the retina in persistent fetal vasculature: emphasis on retinal complications
}

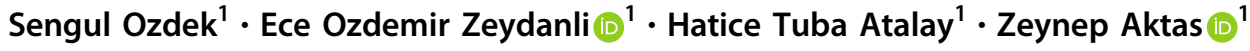

Received: 15 January 2018 / Revised: 4 November 2018 / Accepted: 11 December 2018 / Published online: 6 February 2019

(c) The Royal College of Ophthalmologists 2019

\begin{abstract}
Purpose To identify the characteristics of peripheral retinal anomalies associated with persistent fetal vasculature (PFV) and evaluate the complications and outcomes.

Methods Retrospective, noncomparative case series of patients with PFV who underwent surgery. Type of PFV, presence of peripheral retinal anomalies, extent of the retrolental membrane, complications, and functional and anatomical results were evaluated.

Results Twenty-nine eyes of 28 patients were enrolled in the study: 14 eyes (48.3\%) with anterior PFV, 10 eyes (34.5\%) with posterior PFV, and 5 eyes (17.2\%) with combined PFV. The retina was found to elongate anteriorly as finger-like projections beyond the ora serrata, incorporating into retrolental fibrovascular tissue in $81.8 \%$ of the anterior PFV cases. Cases with more extensive retrolental tissue had a higher risk of retinal complications $(p=0.009)$ and anterior segment complications $(p=0.026)$ than those with localized disease. Leaving the peripheral part of the fibrovascular tissue in place led to complications by later contraction. A total of $35.7 \%$ of the anterior PFV cases had 20/200 or better vision at the final follow-up versus $6.7 \%$ of the cases with posterior involvement. Retinal attachment was achieved in 12 eyes $(80 \%)$ with posterior involvement. Four eyes (13.7\%) resulted in total retinal detachment at final follow-up.

Conclusions The high incidence of peripheral retinal anomalies that are found in anterior PFV patients and their relation to retinal complications warrant careful examination of the ora serrata-ciliary body area. Limbal approach may be preferred as a safer method in cases with an extensive fibrovascular membrane obscuring the view. Leaving the peripheral part of the fibrovascular tissue may result in severe postoperative complications, which should be avoided.
\end{abstract}

\section{Introduction}

Persistent fetal vasculature (PFV), previously known as persistent hyperplastic primary vitreous, is a rare congenital developmental abnormality caused by failure of involution of the primary vitreous and hyaloid vasculature [1]. PFV is a spectrum of disease that includes the remnants of the fetal hyaloid system, extending from the optic disc to the lens to

The topic of this manuscript has been presented in the 20th AVS meeting; however, it has not been previously published elsewhere

Supplementary information The online version of this article (https:// doi.org/10.1038/s41433-019-0345-y) contains supplementary material, which is available to authorized users.

Sengul Ozdek

sozdek@gazi.edu.tr

1 Gazi University School of Medicine, Ankara 06500, Turkey varying degrees. It can be classified as anterior, posterior, and combined forms, according to the affected intraocular structures. Anterior PFV includes the presence of microphthalmia, the retrolental fibrovascular membrane, elongated ciliary processes, or cataract, whereas the posterior subtype consists of an elevated vitreous membrane and a stalk from the optic nerve, retinal fold, or tractional detachment. Nevertheless, most of the cases exhibit features of both and variety of presentations makes PFV challenging for surgical management [2].

The main goals of surgery for PFV are to clear the media to prevent amblyobia and relieve tractional forces to prevent retinal detachment (RD), glaucoma, and phthisis. By releasing the connections of the rigid hyaloidal stalk, the eye has the opportunity to grow and acceptable functional and anatomic results can be achieved [2,3]. However, development of complications such as retinal tears, RD, vitreous hemorrhage, and pupillary obliteration may compromise the success of surgery [4]. Such complications tend 
to occur more often in the presence of anatomical anomalies [5-9].

Here, we report an underdiagnosed and underemphasized anatomical anomaly that may be associated with complications: anterior elongation of the peripheral retina beyond the ora serrata with incorporation into retrolental fibrovascular tissue. Only two studies in the literature previously reported peripheral retinal incorporation into fibrovascular tissue. One is a clinicopathologic study showing that $30 \%$ of the enucleated eyes with PFV had abnormally inserted retina to the pars plicata, in some of which retina was found to be incorporated into the retrolental mass [5]. The other was a single case in which the retina was incorporated into the retrolental tissue causing RD [10]. From our case series, we suggest that this anatomic anomaly may be a much more common finding in anterior PFV patients that warrants extra care during surgery. The purpose of this study was to define and emphasize the importance and higher incidence of this peripheral retinal anomaly in PFV cases while evaluating the outcomes of the surgery.

\section{Subjects and methods}

We undertook a retrospective record review of all consecutive PFV patients who underwent vitreoretinal surgery by one single surgeon (SO) between January 2010 to May 2017 after obtaining an appropriate informed consent. The study followed the principles of the Declaration of Helsinki and was approved by the institutional ethics committee.

Following information from each case recorded, including ages at diagnosis and intervention, gender, associated systemic abnormalities, type of PFV, clinical features and presence of an anatomic anomaly, surgical procedure(s) performed, complications, visual acuity (VA), details of amblyopic therapy, anatomical outcome, and length of follow-up. The extent of fibrovascular tissue covering the back of the lens in the anterior and combined PFV cases was also recorded.

Diagnosis was based on a combination of clinical examination, examination under general anesthesia, and ultrasonography findings. Per Goldberg's descriptions [1], PFV was classified as anterior if a retrolental fibrovascular membrane, elongated ciliary processes, and/or cataract was present, and as posterior when there is tractional retinal detachment (TRD) associated with a stalk from the optic nerve. Cases with features of both were classified as combined PFV.

VA was assessed with age appropriate tests; in younger patients, fixation patterns were recorded using a method modified from that of Zipf [11]:

Fixation patterns: VA

CSM: $>20 / 30$
CSUM: 20/30-20/100

CUSUM: $<20 / 300$

UCUSUM: $<5 / 200$

where $\mathrm{C}$ indicates central; $\mathrm{S}$, steady; M, maintained; US, unsteady; UM, unmaintained; UC, uncentral.

Amblyopia therapy involved patching the better eye for several waking hours every day with placement of a contact lens on the involved eye. The contact lens power was checked by a pediatric ophthalmologist (TA) every 3 months and replaced if necessary.

Statistical analyses were performed using SPSS software, and significance was defined as $p<0.05$. Univariate analysis using $2 \times 2$ tables and a multivariate regression model using ordinary least squares were applied to explore the relationships between baseline characteristics and functional outcomes and to assess risk factors for the development of complications.

\section{Results}

A total of 29 eyes of 28 patients (19 males, 9 females) were identified with PFV during the study period, of which 27 were unilateral and 1 case was bilateral. The median presenting age was 4.5 months ( 1 month-6 years). PFV was an isolated eye pathology without any associated systemic anomaly in all cases. Three patients had a history of prematurity. All patients underwent surgery at a median age of 5 months ( 1 month-6 years). The median length of follow-up was 17.5 months (6 months-5.5 years). Fourteen eyes $(48.3 \%)$ had findings of predominantly anterior PFV, with a retrolental fibrovascular membrane, elongated ciliary processes, or cataract but only a narrow hyaloidal artery remnant without protrusion from the optic nerve or retina. Ten eyes $(34.5 \%)$ had predominantly posterior involvement, including hyaloid remnants and TRD. Five eyes (17.2\%) had features of both anterior and posterior PFV and were classified as having combined PFV.

\section{Functional outcomes}

Five of 14 eyes (35.7\%) with anterior PFV had 20/200 or better vision at final follow-up; 2 achieved a VA of 20/200 according to Snellen or E chart, and 3 had $>20 / 30$ according to fixation patterns (C, S, M). Six had a VA of less than 20/400 to counting finger vision inclusively, and two had light perception (LP) vision. Case 2 had no light perception (NLP) at final follow-up. Of the 15 eyes with posterior involvement (posterior + combined groups), only $1(6.7 \%)$ achieved a VA of 20/200 and one had 20/ 400. Seven had counting finger vision or $<20 / 300$ according to the fixation patterns; three eyes had LP. 
Fig. 1 Case 6: Anterior PFV case in which the retina was continuous with the fibrovascular tissue. When the fibrovascular tissue was cut, there was retina on the other side of the cut tissue, which resulted in a retinotomy (arrow) (a-c). Case 8: Anterior PFV associated with iris, ciliary body, and choroidal coloboma, along with anterior elongation of the retina beyond the ora serrata Prophylactic laser photocoagulation was applied around the coloboma and elongated retina sites $(\mathbf{d}-\mathbf{f})$. Case 10: A dramatic form of anterior PFV with fibrovascular tissue covering the lens entirely, elongated ciliary processes, and tiny retinal elongations are seen $(\mathbf{g}-\mathbf{i})$

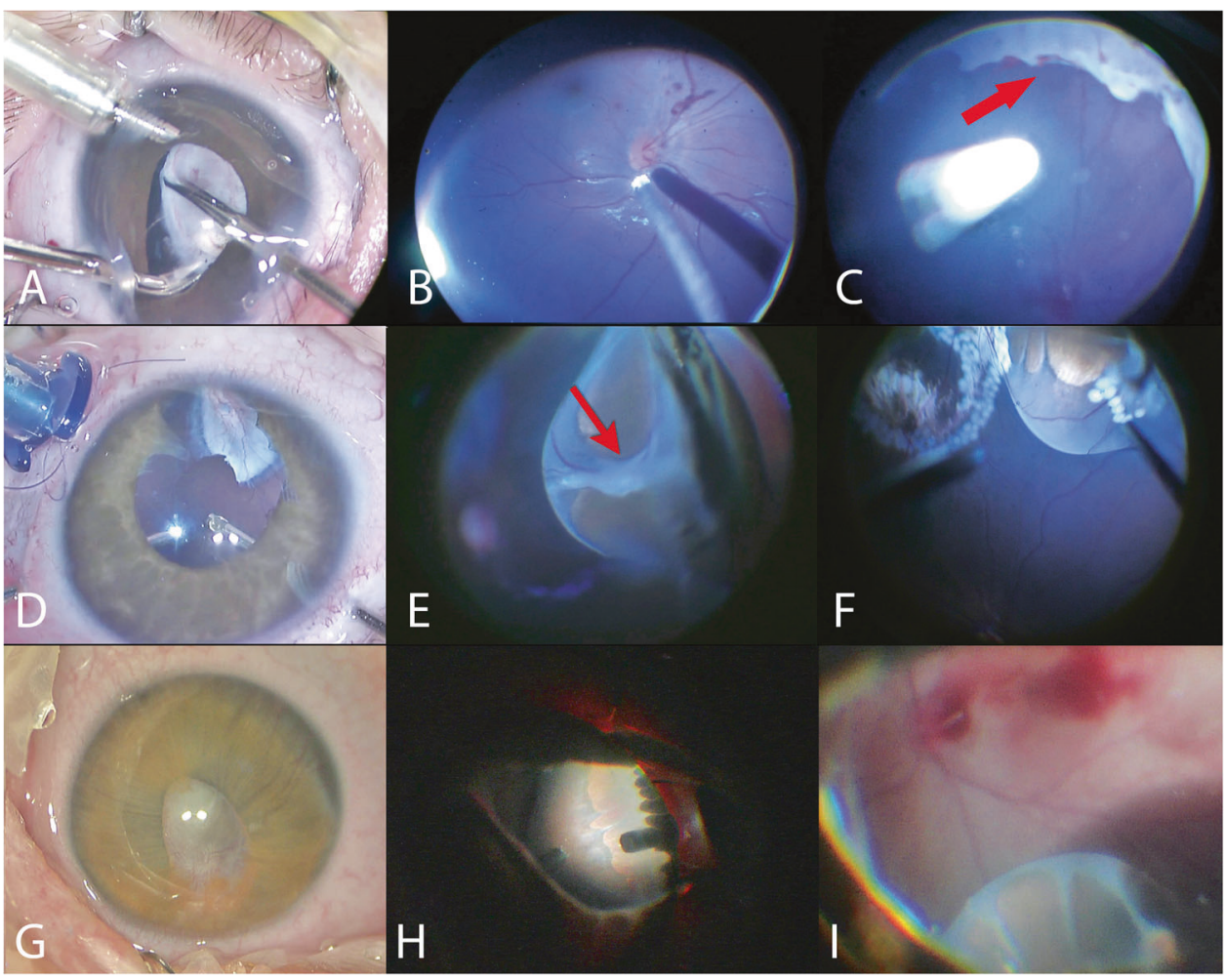

Three eyes had NLP at final follow-up (right eye of case 21 , cases 22 and 27).

The relation of risk factors with poor visual outcomes $(<20 / 200)$ was further analyzed; however, none of the following factors reached clinical significance at multivariate analysis $(R=0.618)$ : posterior involvement $(p=0.06)$, presence of microphthalmia $(p=0.39)$, age at the surgery $(p=0.07)$, length of follow-up $(p=0.69)$, and compliance to amblyobia therapy $(p=0.25)$ (Supplementary Table 1$)$.

\section{Anatomical outcomes}

Eight of 10 eyes with posterior PFV had RD involving the macula, and two had localized peripapillary tractional detachment before surgery. Postoperatively, four eyes $(40 \%)$ had their retinas reattached with significant reversal of retinal dragging and attaining macula reattachment. Four (40\%) had their retinas partially attached with mildly elevated macula. The retina failed to reattach in two eye (right eye of case 21 and case 22). Regarding the combined PFV cases, all five eyes had localized peripapillary detachment without involvement of the macula at the initial examination. The retina was attached in four eyes (80\%); totally detached in one eye (case 27) at final follow-up. Among anterior PFV cases, one resulted in pupillary obliteration and total TRD (case 2). The lens was preserved in all of the posterior cases; however, it was removed in all of the anterior and combined cases.

\section{Complications}

No intraoperative or postoperative complication was noted in the eyes with posterior involvement except for one eye in the combined group (case 27). This eye was complicated with a retinal tear intraoperatively and managed by gas tamponade. However, total RD was documented at final follow-up.

Retinal and/or anterior segment complications were observed in seven eyes $(50 \%)$ in the anterior PFV group. Three eyes were complicated with iatrogenic peripheral retinotomy, all of which were secondary to removal of retrolental fibrovascular tissue intraoperatively (cases 6, 9, and 14). The peripheral retina was observed to be incorporated into the fibrovascular tissue in these cases, hence when the fibrovascular tissue was cut, there was retina on the other side of the cut tissue (Fig.1a-c). Retinal reattachment was achieved by removal of the posterior hyaloid and laser to the retinotomy edges with gas/silicone oil tamponade. These cases led us to explore the ciliary bodyora serrata area in the PFV cases carefully, and peripheral retinal elongations were demonstrated in 9 of 11 eyes with anterior PFV (81.8\%). Earlier cases had not been checked for peripheral retinal anomalies. The peripheral retina was dragged anteriorly, replacing the pars plana totally in some parts, and elongating as finger-like extensions or sometimes circumferentially beyond the ora serrata to become continuous with the retrolental fibrovascular tissue (Fig.1). 


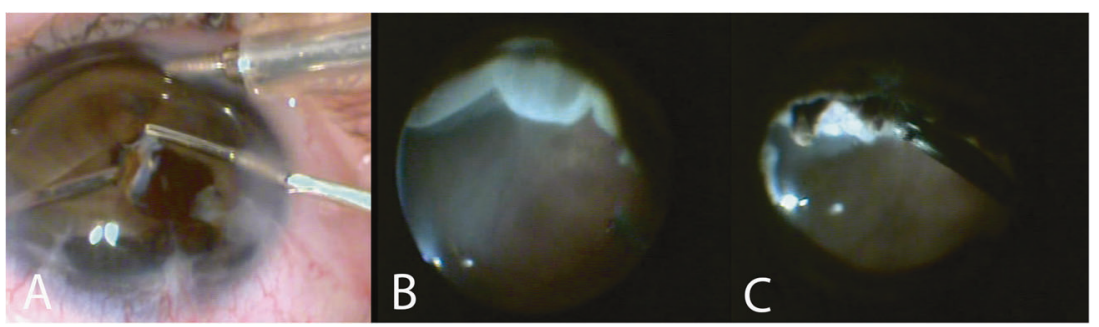

Fig. 2 Case 12: 8-month-old infant with anterior PFV who had a lensectomy and subsequent operations for pupillary obliteration previously; there was iris neovascularization and the pupillary aperture was obliterated by a thick and tenacious membrane that was hard to separate (a). Peripheral retinal detachment was seen (b). The pupillary

Nasal and inferior quadrants were the mostly affected parts. Only one case in the posterior PFV group had peripheral retinal elongations reaching the lens (case 20).

Two eyes with anterior PFV developed peripheral RD postoperatively (cases 2 and 12). These cases had prominent radial iris vessels and the lens extensively covered with fibrovascular tissue. The peripheral part of the fibrovascular tissue was left on purpose during primary lensectomy in both, which later contracted and resulted in peripheral TRD. In case 2, peripheral $\mathrm{RD}$ progressed to total $\mathrm{RD}$ in time with the development of rubeosis iridis and was accepted as inoperable. Case 12 underwent vitrectomy in which peripheral detached retina was found to be continuous in some parts with the contracted fibrovascular membrane (Fig. 2).

Pupillary obliteration was another major postoperative complication, which was documented in three eyes with anterior PFV (cases 5, 12, and 14). In case 5, remaining fibrovascular tissue remnant contracted with $360^{\circ}$ synechia causing pupillary obliteration and pupillary block glaucoma 3 months after primary surgery. He was reoperated immediately and the pupil was reformed by trimming the fibrovascular tissue behind the iris. Three years later, the patient presented with a contracted, narrow but patent pupil with synechia and elevated IOP for the second time. The remaining fibrotic tissues were totally excised, and glaucoma surgery was planned. We observed the retina extending anteriorly in the nasal part, which was unchecked in the primary surgery. Case 12 was referred to our clinic after having had multiple surgeries for a secondary cataract and posterior synechia previously elsewhere. Preoperative ultrasonography yielded peripheral RD besides pupillary obliteration, and vitrectomy was performed as mentioned above (case 12). Case 14 presented with secluded pupilla 8 months postoperatively. We observed fibrotic membranes extending from the peripheral retina, dragging the ciliary body along and causing $360^{\circ}$ synechia; the pupil was reformed with extensive dissection of these membranes. The pupil remained patent, and the retina was attached at follow-up. membrane was continuous with the detached retina in some parts, probably causing traction (c). 360 degree retinotomy, photocoagulation, and silicone oil tamponade resulted in retinal reattachment (not seen in this picture)

Three eyes with anterior PFV were complicated with glaucoma in the postoperative period. One of them was secondary to a pupillary block as described above (case 5). One eye had angle-closure glaucoma due to anterior synechia, which required trabeculectomy (case 4). Case 9 had a temporary IOP elevation, which was controlled with topical antiglaucoma medication until the time of silicone oil removal.

Further analysis of the cases with anterior and combined PFV yielded an association between the extent of lens opacity and overall complications. Eleven of 19 cases (57.9\%) had fibrovascular tissue covering the posterior lens surface entirely; $8(42.1 \%)$ had fibrovascular tissue covering less than half of the lens surface. Six $(31.6 \%)$ had retinal complications; five (26.3\%) had anterior segment complications such as pupillary distortion and glaucoma. All the complicated cases had a dramatic form of the disease with fibrovascular tissue covering the lens entirely. At multivariate analysis, the relationship between the extent of retrolental opacity and retinal and/or anterior segment complications was found to be statistically significant ( $p=$ 0.009 and $p=0.026$, respectively) (Supplementary Table 2).

Patient data for the anterior, posterior, and combined PFV groups are summarized in Tables 1 and 2, respectively.

\section{Discussion}

Reported outcomes of surgery for PFV vary considerably depending on the severity and type of the disease. Purely anterior PFV cases are generally assumed to have better visual potential than those with posterior malformations [12, 13]. However, it has been demonstrated that earlier diagnosis and intervention improve outcomes even for posterior cases [5, 7, 8, 12, 14]. Bosjolie et al. [12]. reported the surgical outcomes of 11 patients with posterior PFV associated with TRD. Ten patients who received surgery before 13 months of age (average age, 4 months) showed 


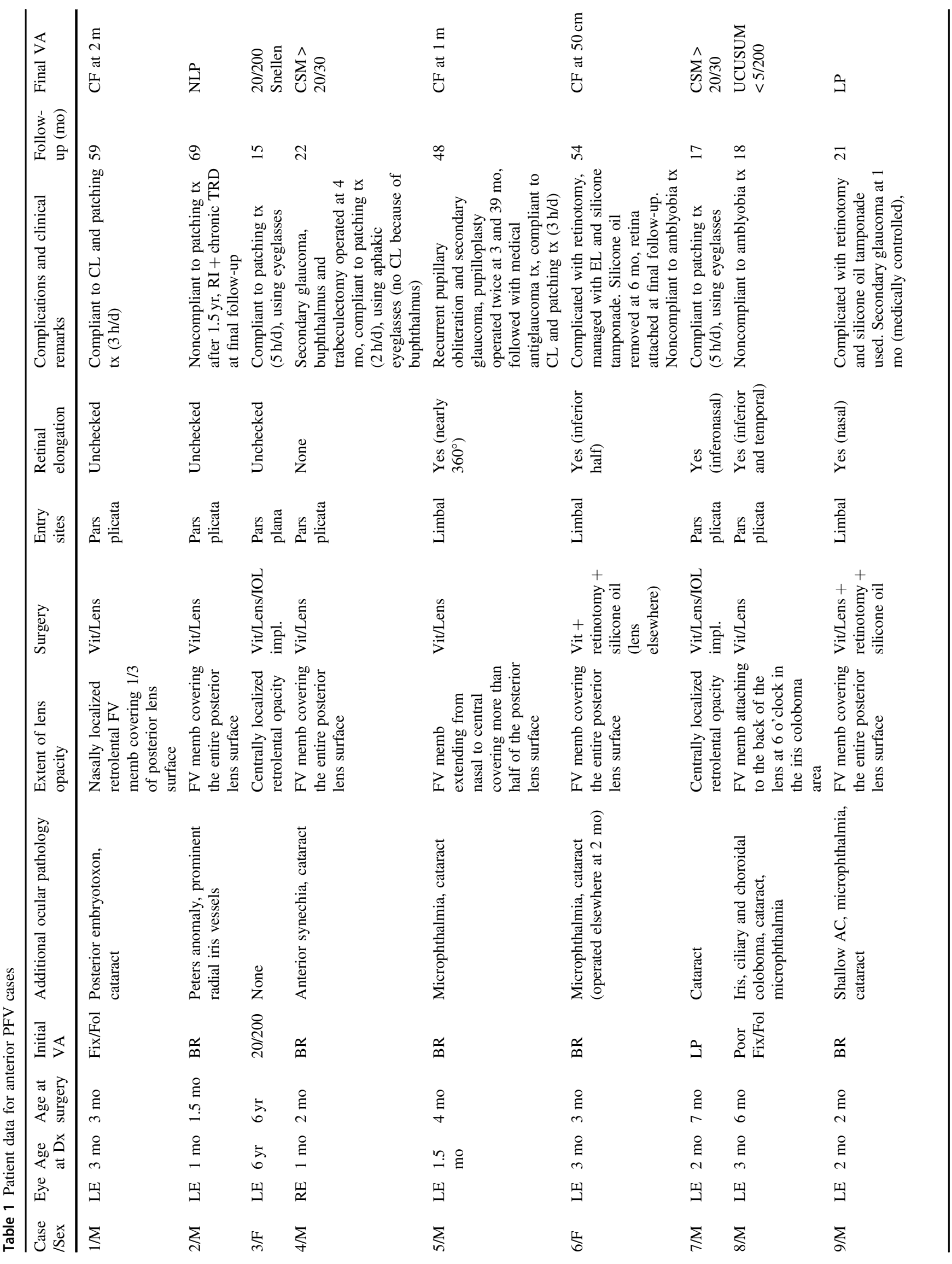




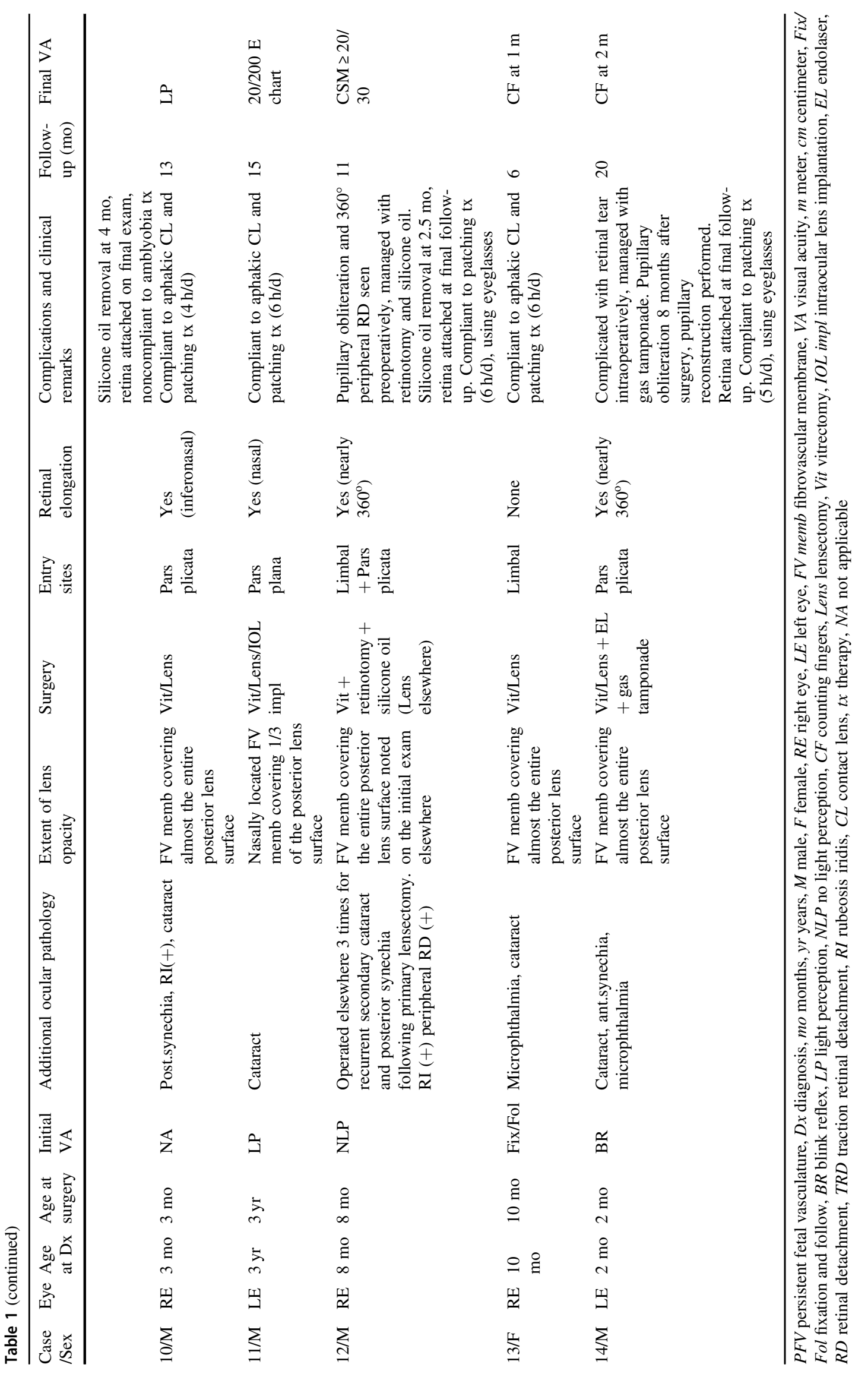




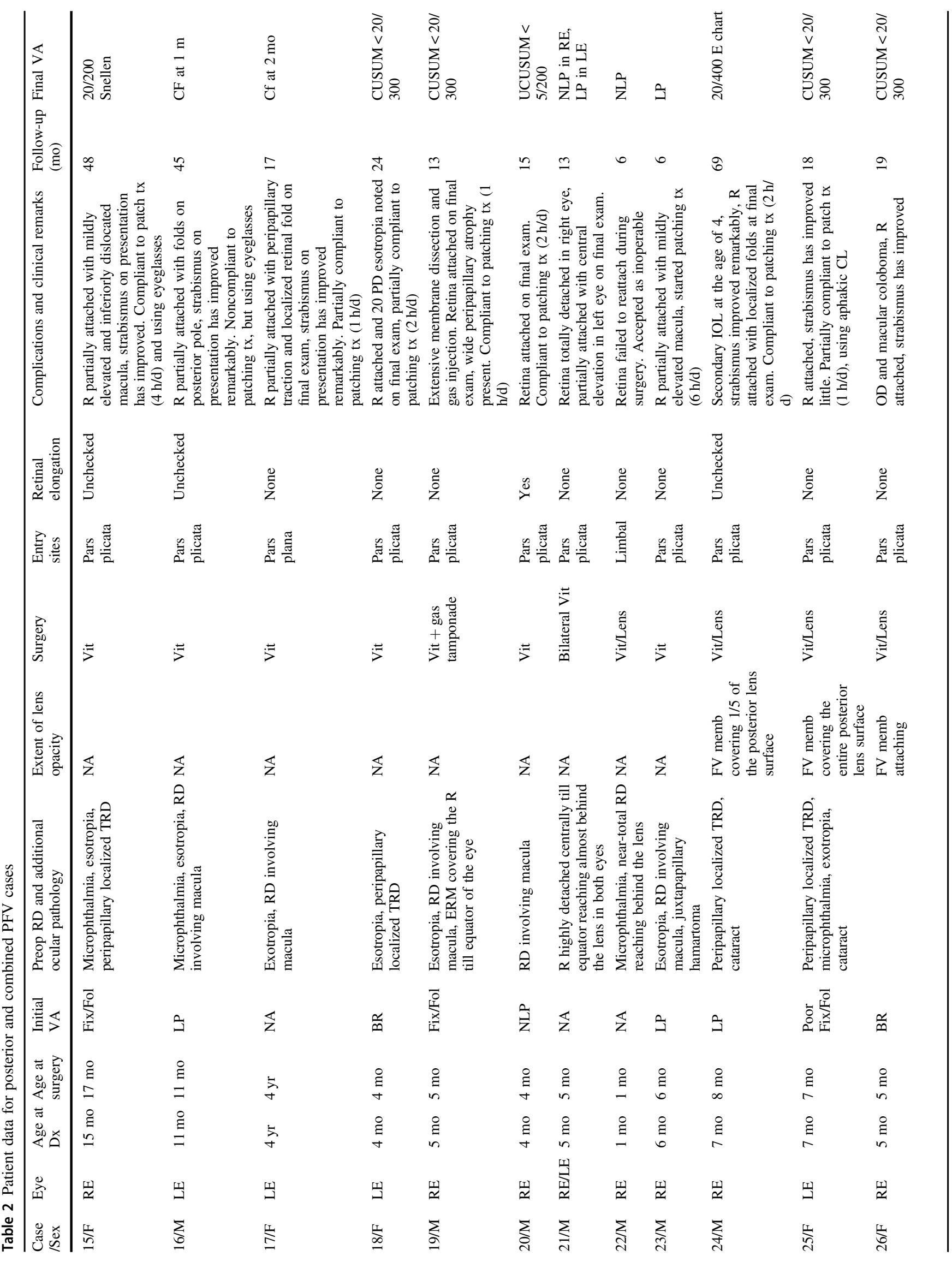




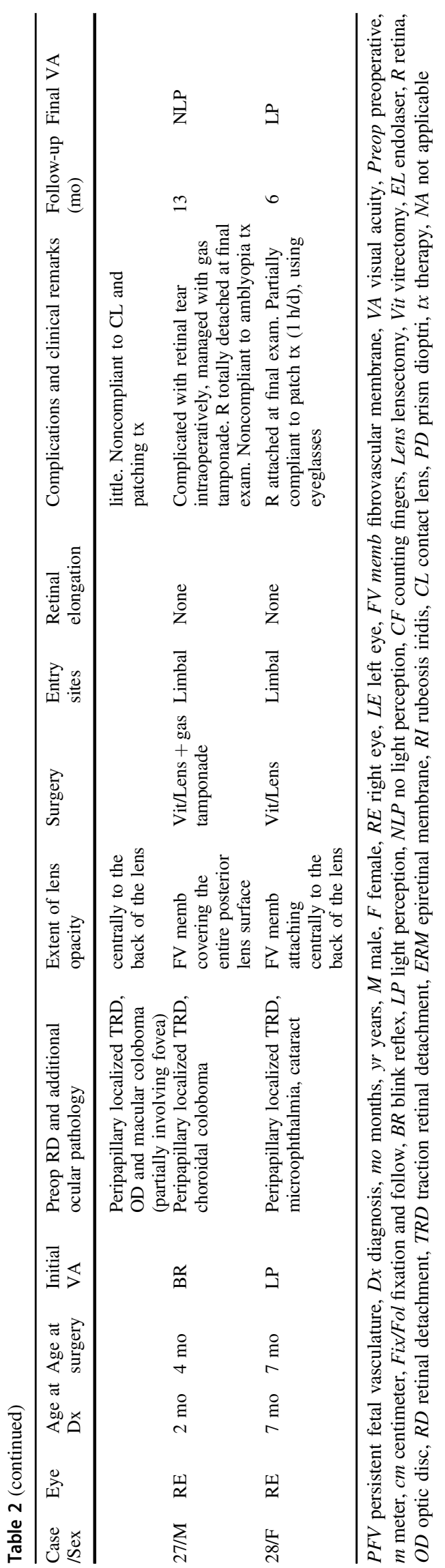

reattachment of retina with significant reversal of retinal dragging, with six of these achieving functional vision of $20 / 800$ or better. The authors noted that the retina has a period of plasticity and emphasized the importance of intervention during this period. Similarly, in a series of 44 eyes that underwent surgery for anterior, posterior and combined PFV, Karaçorlu et al. [13] reported that the median age at the time of surgery was 4 months for the 11 eyes with $20 / 400$ or better postoperative vision, while the average surgical age was 7 months for the 9 eyes with final vision of LP or NLP. Although this difference was not found to be statistically significant, it suggested an association of earlier surgery with better results. However, in contrast to the study by Bosjolie et al. [12], poorer functional and anatomic results were reported in the cases with severe posterior involvement. It was noted that among the eight patients with TRD who underwent surgery at a mean age of 14 months, only three had reattachment of the retina and only one eye achieved a measurable VA of 20/400.

Despite the limitations of our study regarding its retrospective and noncomparative design, the majority of patients achieved vision better than fix-follow in all groups, which showed that even patients with a posterior component of PFV may achieve useful vision after surgery in conjunction with postoperative amblyopia therapy. Eyes with anterior PFV had a higher percentage of better visual outcomes than those with posterior involvement $(35.7 \%$ and $6.7 \%$, respectively). The difference failed to reach statistical significance, which may be attributable to the small sample size. Retinal attachment was achieved in 12 eyes (80\%) with posterior involvement. Four eyes $(13.7 \%)$ resulted in total RD. Two had advanced posterior PFV with near-total RD for which surgical treatment was not effective; two had complications. Our results indicated a significant association between the extent of the lens opacity and complications, which suggests that eyes with more extensive retrolental opacities are more likely to develop complications and should be managed carefully.

The surgical complications led us to define an underdiagnosed and underemphasized peripheral retinal anomaly in PFV. Insertion of the retina anterior to the normal anatomic position, e.g., to the pars plicata, has been previously described in PFV cases in some studies [5-9]. However, literature review yielded only few reports concerning elongation of the retina beyond the pars plicata region. Haddad et al. [5]. reported abnormal insertion of the retina in $30 \%$ of the enucleated eyes with PFV, with only some of them reaching the edges of the retrolental mass in 1978. This was an old histopathological study; therefore, clinical importance of detection and management of this finding was not provided. Karr et al. [10]. reported a single case with incorporation of the retina into the posterior lenticular opacification. Our data indicate that elongation of the retina 
beyond pars plicata and incorporation into the posterior lenticular opacification may be much more prevalent, and may be of prognostic importance. We have documented that $81.8 \%$ of the anterior PFV cases had this anomaly. This finding has not been seen in any of the combined cases in our series; however, one case in the posterior group had peripheral anomaly.

In the presence of this anomaly, total excision of the retrolental fibrovascular membrane or use of pars plicata sclerotomies may increase the risk of inadvertent retinal excision. Furthermore, when the peripheral part of the fibrovascular tissue is left in place, it may contract, resulting in pupillary distortion/occlusion. Since the fibrovascular tissue is continuous with the retina in some parts, its contraction also exerts chronic traction on the peripheral retina causing RD. Iris neovascularization, recurrent hyphema, and glaucoma may develop secondary to chronic $\mathrm{RD}$, which may finally cause a painful eye. Because of these potential complications, fibrovascular tissue should be removed totally and extra care must be taken to avoid iatrogenic damage in the parts that is connected to the retina. We advocate careful investigation of the ora serrata-pars plicata region with indirect ophthalmoscopy, if possible, to be sure that there is no retinal elongation in the planned entry sites. Some clues also suggest the areas of retinal elongation which may guide the surgeon; nasal and inferior parts contiguous to the localized pigmented or fibrovascular plaques are the places where elongated retina parts are most commonly located. Prophylactic laser photocoagulation can also be applied to these areas as necessary.

To our knowledge, this is the first report demonstrating a high incidence of elongation of the retina beyond the ora serrata with incorporation into retrolental fibrovascular tissue in PFV. Given the rates above $80 \%$, extra caution is warranted to avoid complications in anterior PFV cases who may otherwise have good visual potential. It may be safer to use limbal entry in cases with an extensive fibrovascular membrane obscuring view. Finally, leaving part of the fibrovascular tissue should be avoided since this tissue may later contract causing pupillary obliteration, secondary cataract formation, glaucoma, TRD, and iris neovascularization.

\section{Summary}

\section{What was known before}

- Pars plana may be obscured or absent in some cases with persistent fetal vasculature.

- Postoperative complications such as retinal detachment and pupillary obliteration are common in persistent fetal vasculature; however, the etiology is elusive.

\section{What this study adds}

- Majority of the cases with persistent fetal vasculature have anteriorly elongated retinal tissue obscuring pars plana.

- Cases with retinal elongation sites are more prone to complications. Inadvertent retinal damage during surgery or residual fibrous tissue contraction in the postoperative period may be underlying mechanisms.

Funding This research was funded by the Ankara Office of the Turkish Ophthalmology Association. This study was conducted at Gazi University School of Medicine.

\section{Compliance with ethical standards}

Conflict of interest The authors declare that they have no conflict of interest.

Publisher's note: Springer Nature remains neutral with regard to jurisdictional claims in published maps and institutional affiliations.

\section{References}

1. Goldberg MF. Persistent fetal vasculature (PFV): an integrated interpretation of signs and symptoms associated with persistent hyperplastic primary vitreous (PHPV). LIV Edward Jackson Memorial Lecture. Am J Ophthalmol. 1997;124:587-626.

2. Pollard ZF. Persistent hyperplastic primary vitreous: diagnosis, treatment and results. Trans Am Ophthalmol Soc. 1997;95: 487-549.

3. Sisk RA, Berrocal AM, Feuer WJ, et al. Visual and anatomic outcomes with or without surgery in persistent fetal vasculature. Ophthalmology. 2010;117:2178-83 e1-2. https://doi.org/10.1016/ j.ophtha.2010.03.062

4. Cheng LS, Kuo HK, Lin SA, et al. Surgical results of persistent fetal vasculature. Chang Gung Med J. 2004;27:602-8.

5. Haddad R, Font RL, Reeser F. Persistent hyperplastic primary vitreous. A clinicopathologic study of 62 cases and review of the literature. Surv Ophthalmol. 1978;23:123-34. https://doi.org/10. 1016/0039-6257(78)90091-7

6. Reese AB. Persistent hyperplastic primary vitreous. Am J Ophthalmol. 1955;40:317-31.

7. Bird AC, Coleman DJ, Gass JDM et al. Symposium on medical and surgical diseases of the retina and vitreous. Transactions of the New Orleans Academy of Ophthalmology. St Louis (MO): Mosby; 1983. p 228-62.

8. Stark WJ, Lindsey PS, Fagadau WR, et al. Persistent hyperplastic primary vitreous. Surg Treat Ophthalmol. 1983;90:452-7. https:// doi.org/10.1016/S0161-6420(83)34531-0

9. Federman JL, Shields JA, Altman B, et al. The surgical and nonsurgical management of persistent hyperplastic primary vitreous. Ophthalmology. 1982;89:20-4.

10. Karr DJ, Scott WE. Visual acuity results following treatment of persistent hyperplastic primary vitreous. Arch Ophthalmol. 1986; 104:662-7. https://doi.org/10.1001/archopht.1986.01050170052020

11. Zipf RF. Binocular fixation pattern. Arch Ophthalmol. 1976;94:4015. https://doi.org/10.1001/archopht.1976.03910030189003

12. Bosjolie A, Ferrone P. Visual outcome in early vitrectomy for posterior persistent fetal vasculature associated with traction retinal detachment. Retina. 2015;35:570-6. 
13. Karacorlu M, Hocaoglu M, Sayman Muslubas I, et al. Functional and anatomical outcomes following surgical management of persistent fetal vasculature: a single-center experience of 44 cases. Graefes Arch Clin Exp Ophthalmol. 2018;256:495-501.
14. Alexandrakis G, Scott IU, Flynn HW Jr., et al. Visual acuity outcomes with and without surgery in patients with persistent fetal vasculature. Ophthalmology. 2000;107:1068-72. https://doi.org/ 10.1016/S0161-6420(00)00100-7 